\title{
Identités religieuses, appartenance communautaire et participation sociale
}

Anne-Sophie Lamine

\section{OpenEdition}

Édition électronique

URL : https://journals.openedition.org/rdr/1234

DOI : $10.4000 /$ rdr. 1234

ISSN : 2534-7462

Éditeur

Presses universitaires de Strasbourg

\section{Édition imprimée}

Date de publication : 16 novembre 2020

Pagination : 105-121

ISBN : 979-10-344-0073-7

ISSN : 2493-8637

Référence électronique

Anne-Sophie Lamine, «Identités religieuses, appartenance communautaire et participation sociale », Revue du droit des religions [En ligne], 10 | 2020, mis en ligne le 16 novembre 2020, consulté le 02 mai 2022. URL : http://journals.openedition.org/rdr/1234 ; DOI : https://doi.org/10.4000/rdr.1234

\section{(c) $(1)(9$}

La revue du droit des religions est mise à disposition selon les termes de la Creative Commons Attribution - Pas d'Utilisation Commerciale 4.0 International - CC BY-NC 4.0. 


\section{IDENTITÉSRELIGIEUSES, APPARTENANCECOMMUNAUTAIRE ETPARTICIPATION SOCIALE}

\section{Anne-Sophie LAMINE}

Université de Strasbourg / CNRS, Sociétés, Acteurs, Gouvernement en Europe (SAGE)

\section{RésuMÉ}

En partant des difficultés que pose le terme communautarisme (indéfini et disqualifiant), l'approche sociologique permet de proposer des termes descriptifs et des outils pour tenter d'objectiver les phénomènes qualifiés de tels. L'article discute d'abord de l'entre-soi, de l'homophilie et du retournement du stigmate, ainsi que des difficultés propres à la mesure quantitative. La deuxième partie se focalise sur identifications religieuses et appartenance communautaire. Elle en propose une approche pragmatiste qui permet d'en distinguer les modalités et d'en analyser les intensités. Enfin, aucun individu n'étant réductible à son identité religieuse, la dernière partie aborde les modes d'articulation entre les identités composites et la participation sociale.

\section{Abstract}

Building on the difficulties raised by the notion of communitarianism in French (indefinite and discrediting), the sociological approach makes it possible to propose descriptive terms and tools in an attempt to objectivize the phenomena described as such. The article first examines the closed circle, homophilia and the reversal of stigma, as well as the difficulties associated with quantitative measurement. The second part focuses on religious identifications and community belonging. It offers a pragmatist approach that makes it possible to distinguish their modalities and analyze their intensities. Finally, since no individual is reducible to his or her religious identity, the last part addresses the modes of articulation between composite identities and social participation. 
L e communautarisme serait-il la « communauté» des autres ${ }^{1}$ ? L'emploi du terme a connu un tournant quantitatif et sémantique à partir de $1989^{2}$. En effet, une analyse de presse, ainsi que de discours intellectuels et politiques, montre que son usage n'était auparavant ni négatif, ni associé à un groupe ou une époque particulière. C'est à ce moment qu'il devient péjoratif et prend le sens d'une «menace de désintégration sous l'effet des revendications des différentes communautés ethniques ou religieuses ${ }^{3} »$, en France. Il se réfère presque exclusivement à l'islam, aux banlieues et à l'intégration et signifie la crainte du ghetto, du repli sur soi et de l'éclatement de la citoyenneté. Son appropriation résulte aussi de la réception «en biais» du multiculturalisme américain, repoussoir par excellence 4 . Il devient un «ressort rhétorique » pour le débat national lors des campagnes présidentielles à partir de 2007 . «Signifiant malléable», il ne requiert pas de définition, ce qui lui assure un usage très large et consensuel dans le lexique républicain.

Comment dès lors analyser les identités religieuses et l'appartenance communautaire, en prenant au sérieux la question des rapports à la société et au monde commun? Quelle contribution la sociologie peut-elle apporter? Une spécificité de cette discipline est de disposer d'outils qui permettent de porter une grande attention à ce que font les acteurs sociaux au-delà des normes qu'ils défendent ou des discours qu'ils émettent. Cet article privilégie donc une approche pragmatiste.

D'abord, si le terme communautarisme s'avère indéfini et disqualifiant, il s'agit, en sociologue, de proposer des termes descriptifs et des outils pour tenter d'objectiver ces phénomènes. Plusieurs concepts sociologiques s'avèrent pertinents pour analyser ce que l'on qualifie de «repli identitaire »: l'entresoi, l'homophilie ou encore le retournement du stigmate. On portera aussi la réflexion sur les difficultés de la mesure quantitative. La deuxième partie du présent article se focalisera sur identifications religieuses et appartenance communautaire. Elle en proposera une approche pragmatiste qui permet d'en distinguer les modalités et d'en analyser les intensités. Enfin, aucun individu n'étant réductible à son identité religieuse, la dernière partie abordera les modes d'articulation entre les identités composites et la participation sociale.

1. En paraphrasant «l'ethnicité, c'est l'humanité des autres»: D. JutEAu, L'ethnicité et ses frontières, Montréal, Presses de l'Université de Montréal, 1999, p. 165.

2. Période de la première affaire du foulard (1989), de la guerre civile libanaise et du conflit yougoslave: S. Duforx, « Nommer l'autre. L'émergence du terme communautarisme dans le débat français », Socio. La nouvelle revue des sciences sociales, n ${ }^{\circ}$, 2016, p. 163-186.

3. Ibid.

4. Ibid. V. aussi M.-C. GRANJON, «Le regard en biais. Attitudes françaises et multiculturalisme américain (1990-1993)», Vingtième siècle. Revue d'histoire, nº 43, 1994, p. 18-29. 


\section{ENTRE-SOI ET HOMOPHILIES SOCIALES}

\subsection{L'ENTRE-SOI, UNE AFFAIRE DE MINORITAIRES?}

L'entre-soi semble a priori une affaire de minoritaires, pourtant, à y regarder de plus près, il touche divers secteurs de la société. En effet, il est d'abord un "pouvoir sur l'espace ${ }^{5}$ », et, à ce titre, concerne les classes les plus élevées ${ }^{6}$ et plus largement les quartiers résidentiels supérieurs ${ }^{7}$. D'une manière plus générale, au niveau des représentations sociales et de l'ensemble de la société, on constate que l'aspiration à un entre-soi majoritaire est renforcée par l'imaginaire social prégnant de la nation comme une "grande famille», au sein de laquelle les membres «sont censés se ressembler ${ }^{8}$ ». Enfin, si l'entre-soi est un outil de domination pour les uns, il s'avère aussi une forme de résistance pour d'autres. Ainsi, dans les classes populaires, il prend la forme de sentiments de solidarité et d'actes d'entraide ${ }^{9}$ et il s'accompagne souvent de formes de retournement du stigmate ${ }^{10}$ au sein de groupes minorisés.

L'entre-soi des «minoritaires» inquiète, surtout lorsque sa dimension ethnico-religieuse ${ }^{11}$ lui confère une certaine visibilité. Au-delà des représentations liées à cette visibilité ${ }^{12}$ et des études ciblées sur des zones d'entre-soi religieux au sein de certains quartiers populaires, il s'agit de trouver des outils adéquats pour tenter de l'évaluer en le quantifiant. Cependant, le chiffre ne doit pas être considéré comme une donnée objective en soi, dans la mesure où il résulte de la construction d'un indicateur qui peut induire des biais divers, notamment par la polysémie de la question posée, par le sens que sa formulation induit ou par le manque d'options proposées. La polysémie joue aussi son rôle dans l'interprétation des chiffres produits. Si on lit que deux tiers des

5. S. Tissot, «Entre soi et les autres", Actes de la recherche en sciences sociales, $\mathrm{n}^{\circ}$ 4, 2014, p. $4-9$.

6. M. Pinçon, M. Pinçon-Charlot, Les ghettos du Gotha. Comment la bourgeoisie défend ses espaces, Paris, Seuil, 2007.

7. B. Cousin, «Entre-soi mais chacun chez soi. L'agrégation affinitaire des cadres parisiens dans les espaces refondés », Actes de la recherche en sciences sociales, $n^{\circ} 4,2014$, p. 88-101.

8. N. LAPIERRE, Faut-il se ressembler pour s'assembler?, Paris, Seuil, 2020.

9. R. Hoggart, La culture du pauvre, Paris, Minuit, 1970 [1957].

10. E. Goffman, Stigmate. Les usages sociaux des handicaps, Paris, Minuit, 1975 [1963].

11. Rappelons que les sociologues définissent comme ethniques ces processus sociaux de différenciation (ou de fabrication du «nous» versus «eux») qui s'appuient sur des différences réelles ou supposées (incluant des éléments de culture, d'habitus, d'histoire, de langue, de religion ou de couleur de peau), différences mobilisées comme assignation de l'autre ou comme affirmation de soi.

12. V. Amiraux, «Visibility, Transparency and Gossip: How did the Religion of some (Muslims) become the Public Concern of others? », Critical Research on Religion, 4(1), 2016, p. 37-56. 
musulmans souhaitent qu'il y ait plus d'écoles confessionnelles musulmanes, on peut aisément en conclure la prégnance d'une aspiration à l'entre-soi religieux. Par contre, si l'information précédente est immédiatement complétée par le constat que seuls $5 \%$ des musulmans interrogés déclarent qu'ils scolariseraient leurs enfants dans une école confessionnelle musulmane s'ils en avaient la possibilité, l'interprétation change du tout au tout ${ }^{13}$. On comprend, dès lors, que le premier chiffre reflète l'aspiration à des opportunités semblables à celles des autres et à un traitement égalitaire de leur confession. L'étude dont sont tirés ces chiffres montre aussi que, concernant la laïcité, plus de $80 \%$ des musulmans interrogés approuvent la phrase «en France, seule la laïcité permet à des gens de convictions différentes de vivre ensemble». Ces derniers sont également plus positifs (à l'exception de ce qui concerne le logement) à l'égard de la République et de la politique que les autres Français. Finalement, la principale singularité des musulmans est qu'ils «entretiennent avec leur religion une relation plus intense que le reste des Français ${ }^{14} »$.

\subsection{TOUS ENDOGAMES, MAIS LES PLUS HOMOPHILES NE SONT PAS CEUX QUE L'ON PENSE!}

Une autre étude, basée sur l'enquête "Trajectoires et origines» (TeO) de l'INED et l'INSEE, met en évidence que les musulmans ont un taux d'endogamie très voisin de celui des membres d'autres religions ${ }^{15}$. La caractérisation de l'homophilie (qui sont nos amis?) donne aussi une information qui est loin de confirmer l'idée de repli sur le groupe. En effet, lorsque l'on considère les athées-agnostiques, les catholiques et les musulmans, « les plus "communautaires" ne sont pas ceux que l'on croit $^{16} »$ : par comparaison avec les deux autres groupes, les musulmans choisissent plus fréquemment leurs amis en dehors de leur communauté d'orientation religieuse ${ }^{17}$. Dans cette enquête, comme dans la précédente (et a contrario des sondages médiatiques), la question est formulée, non comme un principe, mais en référence à ce que les personnes font réellement.

13. Ces chiffres sont extraits de S. Brouard et V. Tiberj, Français comme les autres? Enquête sur les citoyens d'origine maghrébine, africaine et turque, Paris, Presses de Sciences Po, 2005.

14. Ibid.

15. P. Simon, V. TiberJ, «Sécularisation ou regain religieux: la religiosité des immigrés et de leurs descendants ", Documents de travail INED, no 196, juill. 2013, p. 13-16 (publié in C. Beauchemin, C. Hamel, P. Simon, Trajectoires et origines: enquête sur la diversité des populations en France, Paris, INED, 2016, p. 559-582).

16. Ibid., p. 12.

17. Homophilie religieuse (parmi les amis rencontrés au cours des 15 derniers jours, plus de la moitié sont de la même religion): musulmans: $43 \%$, catholiques: $65 \%$, athéesagnostiques: $51 \%$ : ibid., p. 11. 
Les représentations sociales vont cependant nettement à l'encontre des convergences de pratiques et de valeurs attestées par ces enquêtes sociologiques, comme le montrent des sondages tels que France 2013, les nouvelles fractures ${ }^{18}$. On y lit que $74 \%$ des personnes interrogées considèrent que l'islam n'est pas compatible avec les valeurs de la société française (11\% pour le catholicisme et $25 \%$ pour le judaïsme), et que $80 \%$ des répondants pensent que l'islam cherche à imposer son mode de fonctionnement aux autres ( $21 \%$ pour le catholicisme et $26 \%$ pour le judaisme). Quelles que soient les pratiques réelles des personnes de cultures musulmanes, leur appartenance est souvent confondue avec celle des plus rigoristes d'entre eux. On peine à concevoir qu'une identité religieuse affirmée soit compatible avec une aptitude au pluralisme ${ }^{19}$. Cette différenciation soutenue à l'égard des musulmans (vus comme une culture-religion essentialisée et homogène) qui met l'accent sur l'incompatibilité des valeurs, peut être qualifiée de contreidentification collective et sert d'appui aux discours sur l'identité nationale ${ }^{20}$.

\subsection{JEUNES, MINORITAIRES ET DESCENDANT DE MIGRANTS : PLUS RELIGIEUX}

L'enquête $\mathrm{TeO}$, déjà citée, apporte une autre information pertinente pour notre propos. Parmi les catholiques sondés, $25 \%$ se considèrent comme détachés de la religion, $67 \%$ comme ayant une religiosité modérée et $9 \%$ une religiosité forte. Pour les musulmans, ces chiffres sont respectivement de $4 \%, 47 \%$ et $49 \%$, pour les juifs, $7 \%, 50 \%$ et $43 \%$ et pour les protestants, $16 \%, 54 \%$ et $29 \%$.

Premier constat: les chiffres sont globalement très similaires pour les deux groupes minoritaires juifs et musulmans. Cette similitude est confirmée par la quasi-superposition de leurs courbes de religiosité (fortes et moyennes cumulées) tracées en fonction de l'âge qui indiquent que la religiosité est d'autant plus élevée que les individus sont jeunes. Deuxième constat, la religiosité des juifs et des musulmans est nettement plus élevée que celle des

18. Sondage IPSOS - Le Monde - Fondation Jean-Jaurès - Cevipof, «Les nouvelles fractures», janv. 2013.

19. Alors que les observations contredisent cette hypothèse d'incompatibilité, comme on le verra plus loin.

20. F. Lorcerie, K. Romhild-BenkaAba, N. Tietze, «Politisation autour de l'islam et légitimation des ordres politiques nationaux en Europe», in F. FORET (dir.), L'espace public européen à l'épreuve du religieux, Bruxelles, Éd. Université de Bruxelles, 2007, p. 179-206; cette contre-identification se joue sur trois registres: ethno-culturel, valeurs politiques et menace vitale (terrorisme).

21. P. Simon, V. TiberJ, «Sécularisation...», op. cit., p. 10. 
catholiques, les protestants se situant dans une position intermédiaire. Troisième constat, si l'on extrait un sous-groupe parmi les catholiques, constitué de ceux d'origine non européenne (principalement des territoires d'outremer et d'Afrique subsaharienne), le taux de forte religiosité s'élève de $9 \%$ à $29 \%$ (et celui des autres catholiques baisse à $7 \%$ ). Indépendamment de la religion, cela démontre l'effet de la migration sur le niveau de religiosité. Un autre résultat de l'enquête confirme ce point: les descendants d'immigrés ont une religiosité nettement plus élevée que la population «majoritaire». Ce fait est d'autant plus marqué que les personnes sont jeunes, alors que chez les majoritaires, au contraire, la religiosité augmente avec l'âge.

La situation intermédiaire du protestantisme appelle un commentaire spécifique $^{22}$. En effet, ceux qui se disent protestants sont en majorité luthériens ou réformés (56\%) et, pour approximativement un tiers (30\%), de sensibilité évangélique (évangéliques, pentecôtistes ou charismatiques), 14 \% ne se prononçant pas. Cependant, si l'on se restreint aux protestants présents au moins une fois par mois au culte, les évangéliques deviennent majoritaires: 55 \% sont évangéliques, pentecôtistes ou charismatiques, 37 \% luthériens ou réformés, et $8 \%$ indéterminés. En outre, on observe aussi un effet de religiosité plus élevée chez les jeunes de sensibilité évangélique.

Globalement, les minoritaires (religieusement et/ou issus des migrations) sont donc plus religieux, et ce d'autant plus qu'ils sont jeunes, et ils transmettent beaucoup mieux la religion à leurs enfants que les majoritaires.

\subsection{ISLAM IS BEAUTIFUL, OU LE RETOURNEMENT DU STIGMATE}

Les sociologues des mouvements sociaux soulignent le rôle que peut prendre la dimension identitaire au sein de groupes stigmatisés (ethniques, sexuels...). En effet, ces derniers visent par leurs discours et leurs actions à contester l'image négative prévalant à leur égard et à la modifier en une image plus favorable ${ }^{23}$. Les recherches sur les jeunes musulmans sont tout à fait congruentes avec cette observation, et décrivent toute une gamme de préoccupations et d'attitudes visant à corriger cette image négative. D'un côté, des jeunes musulmans très pieux, le plus souvent issus de familles de classes populaires, pour lesquels la religiosité orthodoxe prend une grande

22. Ce paragraphe s'appuie sur: J.-P. Willaime, «Les protestants de France au miroir du sondage IFOP de 2010 » et «Les pasteurs de France au miroir du sondage IFOP de 2010 », in S. FAth, J.-P. Willaime (dir.), La nouvelle France protestante: essor et recomposition au XXI ${ }^{e}$ siècle, Genève, Labor et Fides, 2011, p. 399-437.

23. L. Mathieu, Comment lutter? Sociologie et mouvements sociaux, Paris, Textuel, 2004, p. 141. 
place, affirment souvent leur islamité avec fierté, dans un processus de renversement du stigmate ${ }^{24}$. Ce faisant, ils manifestent une vision critique de la société (stigmatisation, impérialisme, manque de spiritualité), tout en s'en revendiquant comme membres à part entière. Dans ce sens, on peut très justement parler d'une «inclusion négociée ${ }^{25}$ » dans une société où tout n'est pas négatif et dans la transformation de laquelle une part significative d'entre eux s'engage. D'un autre côté, d'autres jeunes personnes pieuses sont aussi soucieuses de rectifier l'image de leur religion, mais de manière beaucoup moins critique vis-à-vis de la société $e^{26}$.

Le retour du stigmate, pour des croyants d'origine immigrée, ou la fierté de montrer sa religiosité, y compris ultra-orthodoxe, n'est évidemment pas l'apanage de musulmans, comme le montre l'observation des Églises évangéliques ethniques ${ }^{27}$ ou encore de la mise en scène publique de Hanouca dans les grandes villes d'Europe par les Loubavitchs ${ }^{28}$.

\section{UNE LECTURE PRAGMATISTE DE L'IDENTITÉ RELIGIEUSE}

\subsection{UNE CONFUSION FÂCHEUSE, CROYANCE INTENSIVE ET INTRANSIGEANTISME}

La représentation sociale partagée du croire intensif suppose que l'intensification de la religiosité va de pair avec celle de l'intransigeantisme. Cette affirmation s'appuie sur des cas réels de personnes à la fois très religieuses et très dogmatiques, rigides, peu tolérantes à la différence. De tels cas peuvent s'avérer problématiques dans les relations sociales: que ce soit au travail

24. V. par ex.: M. KHEDimellah, « Tabligh ou l'islam apostolique au masculin dans les quartiers impopulaires de France», in C. CosséE, E. LAdA, I. RigOni (dir.), Faire figure d'étranger, Paris, Armand Colin, 2004, p. 261-276. Cela est très net aussi dans les mobilisations «en tant que musulman», notamment dans les quartiers ou contre l'islamophobie: J. TALPIN, J. O'Miel, F. Frégosi, «Introduction. Se mobiliser en tant que musulman. Condition minoritaire et engagement politique», in J. TAlPin, J. O'Miel, F. Frégosi (dir.), L'islam et la cité: engagements musulmans dans les quartiers populaires, Lille, Presses universitaires du Septentrion, 2017, p. 27-59 (spéc. p. 32).

25. A. Villechaise, L. Bucaille, «L'affirmation religieuse des jeunes musulmans», Revue européenne des sciences sociales, $\mathrm{n}^{\circ}$ 56-2, 2018, p. 107-131.

26. J. Jouili, Pious Practices and Secular Constraints. Women in the Islamic Revival in Europe, Stanford, Stanford University Press, 2015, p. 187-199.

27. S. FATH, «La gestion du stigmate, entre local et global: trois megachurches afro-caribéennes à Paris», in L. Endelstein, S. Fath, S. Mathieu (dir.), Dieu change en ville. Religion, espace, migration, Paris, L'Harmattan, 2010, p. 115-135.

28. L. Endelstein, «Lumières sur la ville. Les fêtes de Hanoucca entre action missionnaire transnationale et appartenance événementielle», Archives de sciences sociales des religions, $\mathrm{n}^{\circ} 177,2017$, p. 51-71. 
ou au sein d'institutions, par exemple lorsqu'un homme refuse d'avoir une interlocutrice féminine, comme conseillère pénitentiaire ${ }^{29}$ ou à l'emploi. Les observations montrent que, la plupart du temps, cette relation est cependant bien plus complexe. Dans de nombreux cas, des personnes sont à la fois très croyantes (y compris visiblement) et sensibles à l'altérité. Elles composent avec les autres, avec la société et y contribuent. Elles ne sont donc pas intransigeantes. Inversement, des personnes peu ou pas pratiquantes peuvent être intransigeantes.

Cette distinction a initialement été développée dans un ouvrage de référence sur la diversité du catholicisme, en lien avec les convictions politiques. L'auteur souligne cette confusion fréquente entre «intransigeantisme» et «intégralisme»: «Ces deux termes [correspondent à] deux réalités tout à fait différentes. L'intransigeantisme désigne le refus de transiger avec des données culturelles extérieures au modèle catholique originel, l'intégralisme le souci d'englober la totalité des actes et des pensées dans la référence religieuse, de ne pas reléguer le religieux dans un espace et un temps précis, de ne pas séparer les attitudes religieuses de toutes les autres attitudes exprimées par le sujet ${ }^{30}$.» En plus des deux combinaisons spontanément envisagées: faible religiosité sans intransigeantisme et intensité religieuse avec intransigeantisme, on peut donc ajouter deux nouvelles combinaisons: intensité religieuse sans intransigeantisme et faible religiosité avec intransigeantisme.

Cette distinction s'avère pertinente pour tout groupe religieux ${ }^{31}$. Elle permet d'envisager des cas contre-intuitifs. Dans le cas de l'islam, ce sont par exemple ces «citoyennes pieuses $^{32}$ » de confession musulmane, autant attachées à leur pratique religieuse, incluant souvent le port du foulard, qu'à leurs place et rôle de citoyennes. Elles manifestent un sens étendu du bien commun, qui englobe la société et l'humanité dans leur ensemble, et une intersubjectivité large, une capacité de comprendre et de se mettre à la place de personnes d'autres convictions. Inversement, même si cela est moins documenté, des personnes peuvent être intransigeantes, au nom de

29. V. par ex. R. SARG, A.-S. LAMINE, «La religion en prison. Norme structurante, réhabilitation de soi, stratégie de résistance», Archives de sciences sociales des religions, $\mathrm{n}^{\circ} 153$, 2011, p. 85-104 (spéc. p. 99).

30. J.-M. Donegani, La liberté de choisir. Pluralisme religieux et pluralisme politique dans le catholicisme français contemporain, Paris, Presses de Science Po, 1993, p. 406; sur le catholicisme intégral, V. É. Poulat, Intégrisme et catholicisme intégral. Un réseau secret international antimoderniste: "La Sapinière" (1909-1921), Paris, Casterman, 1969.

31. Il faudrait néanmoins y ajouter la distinction de diverses formes d'intransigeantisme (LAmine, travaux en cours).

32. J. Joulli, Pious Practice..., op. cit., p. 187-199. 
leur religion, tout en étant peu (ou pas) religieuses ${ }^{33}$. Dans ce second cas, l'identité religieuse, bien que peu ou pas pratiquée, est mobilisée comme une ressource d'opposition à la société, par exemple dans cette catégorie de jeunes musulmans «littéralistes, mais peu [ou pas] pratiquants ${ }^{34}$ », rencontrés en quartiers populaires, notamment parmi ceux qui sont (ou ont été) plus ou moins délinquants. Ils montrent une «idéalisation du religieux [islamique] », comme «la solution à tous leurs maux, même s'ils ne sont pas pratiquants » et admirent les salafistes, les «vrais musulmans».

L'intransigeantisme correspond donc à une réduction de la place de l'intersubjectivité dans la relation à autrui (notamment à l'égard de ceux qui ont d'autres convictions et pratiques) tout comme de la vision du bien commun ${ }^{35}$. Si l'intransigeantisme s'allie à l'intégralisme, le système religieux ou convictionnel tend à devenir la référence unique (pour toutes les questions, tous les aspects de la vie et des relations aux autres). Cela se manifeste notamment dans le dualisme des discours opposant le groupe religieux à la société, dans une recherche de religion pure, débarrassée des scories de la culture, et qui n'est l'apanage d'aucun courant religieux ${ }^{36}$. Certaines réactions virulentes ou violentes face aux usages littéraires, artistiques ou publicitaires de figures ou de scènes religieuses montrent que la culture ambiante est alors perçue par certains croyants comme un «blasphème permanent ${ }^{37}$ ».

\subsection{CE À QUOI NOUS TENONS}

$\mathrm{Au}$ moment des débats sur les signes religieux en 2003, on a entendu des responsables de sécurité intérieure, d'établissements scolaires ou de services du personnel affirmer que prendre un jour de congé pour la fête de l'Aid était une forme de revendication politique ${ }^{38}$. Telle affirmation place la

33. T. YiLDiz, Qui sont-ils? Enquête sur les jeunes musulmans de France, Paris, Éd. du Toucan, 2016.

34. Ibid., p. 19-27.

35. Dans les cas les plus extrêmes, l'intersubjectivité et le bien commun se réduisent au petit groupe d'«élus». Le bien commun peut aussi se trouver projeté dans un futur eschatologique.

36. J. Roeland, S. Aupers, D. Houtman, M. De Koning, I. Noomen, «The Quest for Religious Purity in New Age, Evangelicalism and Islam », Annual Review of the Sociology of Religion, 1, 2010, p. 289-306.

37. O. Roy, La sainte ignorance. Le temps de la religion sans culture, Paris, Seuil, 2008; I. SAINTMartin, "Christ, Pietà, Cène, à l'affiche: écart et transgression dans la publicité et le cinéma », Ethnologie française, $\mathrm{n}^{\circ}$ 1, 2006, p. 65-81.

38. Argument encore plus massivement entendu à propos du maillot de bain couvrant (burkini) en août 2016. 
pratique musulmane hors équivalence (tenir à célébrer Pâques ou Noël ne donnant évidemment pas lieu à une lecture similaire). L'expérience religieuse, l'attachement à une fête, à une manière de se nourrir ou de se vêtir, est rarement appréhendée comme une expérience, une pratique à laquelle on tient, tout comme d'autres Français tiennent aux fêtes d'anniversaire ou à leur régime végétarien. Le sens commun ne fait pas de place à ces attachements.

Les questions que pose le religieux dans l'actualité conduisent à inclure la question des valeurs et des attachements. Il n'y a certes rien de neuf à souligner que l'éthique est une dimension fondamentale du religieux, par son rôle dans l'identification au religieux ${ }^{39}$ ou lorsque ce dernier est mobilisé pour remettre en ordre l'existence ${ }^{40}$. L'approche pragmatiste ${ }^{41}$ permet cependant d'articuler la dimension éthique au rôle de l'attachement (dimension émotionnelle-expérientielle), en considérant «ce à quoi les gens tiennent». Si l'on envisage les valeurs comme «ce à quoi l'on tient», elles sont le produit de l'activité intelligente, en lien avec l'expérience, qui inclut des composantes émotionnelles et intersubjectives. Cette perspective distingue analytiquement deux niveaux: l'appréciation immédiate (plus émotionnelle, expérientielle) et le jugement, l'évaluation (plus cognitif, rationnel). Des croyants d'une association musulmane ou d'une Église évangélique défendent la valeur d'égalité, mais plus précisément, ils tiennent à des conditions d'égale dignité pour l'exercice de leur culte et agissent en conséquence.

\subsection{TROIS MODALITÉS DE L'IDENTIFICATION RELIGIEUSE OU DU CROIRE EN ACTE}

L'attention à "ce à quoi les gens tiennent» permet dès lors de distinguer trois modalités de la religiosité vécue, dans une perspective sociologique pragmatiste (deweyenne), et présente en outre l'intérêt de faciliter la désingularisation de l'acte de croire. Croire religieusement, c'est «tenir à» des expériences de construction de soi, de dépassement de soi et de fabrication du commun, avec une référence à une tradition religieuse particulière ${ }^{42}$.

39. D. Hervieu-Léger, Le pèlerin et le converti, Paris, Flammarion, 1999.

40. N. Tietze, Jeunes musulmans de France et d'Allemagne: les constructions subjectives de l'identité, Paris, L'Harmattan, 2002.

41. J. Dewey, La formation des valeurs (éds et trad. A. Bidet, L. Quéré, G. Truc), Paris, La Découverte, 2011 [1918, 1925, 1939, 1944].

42. A.-S. Lamine, Identités religieuses et monde commun. Penser les idéaux, les attachements et la participation sociale avec John Dewey, Paris, L'Harmattan, 2018; «Religion as Experience. Dialogue with John Dewey», Social Compass, 65/5, 2018, p. 667-683. 
La première modalité du croire en acte est celle de la construction de soi. Cette expérience, sensible et corporelle, inclut des pratiques religieuses, mais aussi des actes de transmission ou de contemplation. L'expérience peut être définie comme «une forme de vitalité et d'interaction avec le monde, captant totalement l'attention et menant à un sentiment d'unité intérieure et avec le monde ${ }^{43} »$. On peut d'abord «vivre une expérience ${ }^{44} »$, ce qui implique une attention soutenue à l'instant présent (notamment dans l'attitude contemplative). On peut aussi effectuer un «travail sur soi», qui implique davantage une discipline personnelle (prière, lecture, jeûne...), tout en s'appuyant sur des interactions et des ressources disponibles résultant de transmissions et de validations sociales. Signalons des analogies. Cet effort sur soi prend du temps et de l'attention. Il n'est pas si différent, dans sa nature, d'une pratique assidue de la musique ou d'un sport par exemple.

La deuxième modalité du croire en acte qui s'exprime dans le religieux, mais aussi dans l'expérience séculière, renvoie au dépassement de soi ou à l'aspiration à s'élever au-dessus de la satisfaction de ses besoins quotidiens. Le symbolique est nécessaire à l'individu, notamment pour exprimer ce qui le «dépasse». Mais le religieux ne s'y réduit pas. Dans sa mise en acte, le dépassement de soi passe par des idéaux, définis comme des valeurs auxquelles on tient et pour lesquelles on s'engage dans la durée ${ }^{45}$, et dans le cas des croyants au nom de leur foi. Ces idéaux sont la justice, la solidarité, l'environnement ou la paix, etc.

La troisième modalité du croire en acte est celle d'être ensemble ou de faire communauté. Si la dimension émotionnelle est toujours présente dans l'action et l'interaction, elle l'est ici de manière particulièrement forte, notamment dans les rituels ou dans les grands rassemblements, mais aussi dans les formes de relations chaleureuses et les solidarités pratiques entre membres du groupe. Cependant, les choses sont plus complexes qu'elles n'en ont l'air. On constate qu'avec le groupe auquel les «croyants» tiennent, dont ils sont (très) solidaires, les désaccords sont fréquents, ils sont la norme plutôt que l'exception. La critique interne, portant sur les rapports de pouvoir internes et notamment de genre, mais aussi sur les formulations du «bien croire» ou du «bien agir», bien que souvent discrète est pourtant omniprésente. Tenir (y compris très fort) à sa communauté tout en la critiquant sur certains

43. A.-S. Lamine, Identités religieuses..., op. cit., p. 73.

44. J. Dewey, L'art comme expérience (trad. J.-P. Cometti et al.), Paris, Gallimard, 2010 [1934].

45. A.-S. Lamine, Identités religieuses..., op. cit., p. 129, en s'appuyant sur J. Dewey, Une foi commune (trad. P. Di MASCIO), Paris, La Découverte, 2011 [1934]. 
points, c'est ce qu'exprime la notion de «solidarité sans consensus ${ }^{46}$ » qui est une caractéristique constante du rapport au religieux. Enfin, notons que, tout comme des croyants «font» communauté, les acteurs sociaux inquiets du religieux «font» «anti-communauté », avec un rôle non moins important des émotions, notamment en qualifiant les religieux de "communautaristes». En effet, comme on l'a déjà vu, la perception ordinaire du religieux oppose l'idée des communautés à celle du monde commun, ce qui nous invite à nous interroger sur les modes et les possibilités de participer au bien commun à partir d'identités particulières.

\section{IDENTITÉS COMPOSITES ET PARTICIPATION SOCIALE}

Au-delà des appartenances religieuses et des craintes diverses qu'elles suscitent, il s'agit aussi de comprendre les articulations entre le monde commun et ces identités particulières. L'idée de communauté évoque souvent celle de différenciation, voire de repli. Pourtant, le caractère composite des identités ne permet-il pas de vivre plusieurs appartenances, plusieurs attachements? L'attachement à des formes de croire religieux va-t-il (forcément) à l'encontre d'une identification à la société globale et d'un souci pour le monde commun? Les processus de différenciation sont-ils dès lors les seuls observables?

\subsection{IDENTITÉS COMPOSITES}

Les identités des individus sont intrinsèquement composites, dans la mesure où ces derniers se situent au croisement de diverses sphères ${ }^{47}$ : familiale, amicale, confessionnelle ou convictionnelle, intellectuelle, de genre, de profession, de culture, de voisinage, d'engagement associatif, etc. En outre, la vision d'instantanés de pratiques croyantes peut faire négliger la temporalité des rapports au croire, donc les «carrières croyantes» (tout comme l'on parle de carrières militantes). En effet, l'observation sur la durée montre que l'engagement religieux varie selon les périodes de la vie. Un engagement très intensif ne dure souvent que quelques années et s'atténue ensuite avec modération, prise de distance ou engagement plus social. Cela s'observe, par exemple, parmi de jeunes chrétiens évangéliques conservateurs

46. La formule vient de Kertzer, qui l'applique aux cérémonies politiques: D. KerTzER, Ritual, Politics and Power, New Haven, Yale University Press, 1988.

47. G. Simmel, «Le croisement des cercles sociaux», in Sociologie. Étude sur les formes de la socialisation. Paris, PUF, 1999 [1908], p. 407-452. 
très engagés ${ }^{48}$ ou des membres militants du mouvement de réislamisation Tabligh $^{49}$, notamment au moment où ils se stabilisent professionnellement et fondent une famille.

\subsection{LA PARTICIPATION SOCIALE : LA DÉMOCRATIE COMMENCE DANS LE VOISINAGE}

La perspective pragmatiste de la démocratie est celle d'une «coopération sociale», qui peut faciliter la résolution des problèmes sociaux ${ }^{50}$. Ce concept de participation sociale comme cœur de la démocratie prend une acuité particulière dans un contexte de pluralité culturelle, ethnique et religieuse. Dewey définit ainsi l'«idée démocratique»: «le fait [pour l'individu] de prendre part de manière responsable, en fonction de ses capacités, à la formation et à la direction des activités du groupe auquel il appartient, et à participer en fonction de ses besoins aux valeurs que le groupe défend ${ }^{51}$ ». Chaque individu étant membre de plusieurs groupes, les valeurs sont celles de l'école des enfants, de l'association du quartier, du groupe professionnel, de la congrégation, etc. Dans cette perspective, il n'y a pas compétences prérequises à la participation, c'est en faisant que l'individu acquiert les compétences citoyennes.

Cette participation sociale s'effectue avec une perspective d'un bien commun plus large que celui d'un unique groupe d'appartenance. Dans ce sens, elle relève d'un domaine que l'on peut qualifier de «prépolitique ${ }^{52} »$. Elle s'exerce le plus souvent dans un domaine de proximité, ce qui fait écrire à Dewey que «la démocratie doit commencer à la maison, et sa maison, c'est la communauté de voisinage ${ }^{53} »$. La «communauté» (scolaire, de quar-

48. E. Buchard, «Éduquer et être éduqué dans la foi évangélique», in J. Stolz, O. Favre, C. GACHET, E. BuCHARD, Le phénomène évangélique, analyse d'un milieu compétitif, Genève, Labor et Fides, 2013, p. 184.

49. M. Khedimellah, «Jeunes prédicateurs du mouvement Tabligh», Socio-anthropologie, $\mathrm{n}^{\mathrm{o}} 10,2001$ : https://journals.openedition.org/socio-anthropologie/155 [consulté le 17 juin 2020].

50. J. Dewey, Le public et ses problèmes (trad. J. ZasK), Paris, Gallimard, 2010 [1927], p. 313. L'approche pragmatiste se distingue d'autres approches, notamment de celle de Habermas, dans la mesure elle n'est pas un modèle de communication et consultation, A. HonNETH, «Democracy as Reflexive Cooperation. John Dewey and the Theory of Democracy», Political Theory, 26/6, 1998, p. 776-777.

51. Ibid., p. 242.

52. Honneth, en lisant Dewey, souligne que la vitalité des publics démocratiques présuppose une forme d'intégration sociale par une «conscience commune de l'association prépolitique des citoyens», qui s'appuie sur leurs actions coopératives et leur poursuite de buts communs: A. HonNeTH, «Democracy...», op. cit., p. 776-779.

53. J. DeWey, Le public..., op. cit., p. 317. 
tier, d'usagers, de patients, etc.) existe par l'action conjointe et le fait que les conséquences de cette action sont considérées comme un bien par les personnes qui y prennent part ${ }^{54}$. Dans cette perspective, la "grande communauté» n'est autre que la démocratie: «la conscience claire de la vie commune, dans toutes ses implications, constitue l'idée même de démocratie $^{55} »$. La démocratie passe par la fabrication du commun, par le désir et l'effort d'y contribuer, de manière individuelle et collective.

En laissant de côté certains accents normatifs (mélioristes) des écrits de Dewey, on y trouve des concepts pertinents pour interroger et analyser la fabrication du monde commun, pour observer comment il se fabrique dès lors que des acteurs sociaux, bien qu'agissant de manière très locale (environnement, climat, quartier, école) ou en étant ancrés dans une identité particulière (religion, ethnicité, habitat spécifique...), le font avec une conscience du bien commun, avec une perspective plus large que celle de leur groupe restreint ${ }^{56}$. Ces outils s'avèrent très complémentaires de ceux de la sociologie des relations interethniques qui, en se focalisant sur les (réels) processus de différenciation sociale, n'éclaire pas les processus inverses de dé-différenciation.

\subsection{FAIRE (OU ÊTRE INTERDIT DE FAIRE) DU COMMUN À PARTIR DU PARTICULIER}

L'idée de participation sociale mène à deux questions: peut-on fabriquer du commun à partir de positions particulières et y a-t-il des prérequis imposés à l'individu (particulier) pour autoriser sa participation sociale? Deux exemples empiriques étayeront ces deux pistes de réflexion.

L'analyse d'un média musulman français en ligne, Saphirnews.com, donne un matériau pour travailler sur la première tension. Ce média est fondé suite à la prise de conscience d'un problème commun: la perception négative de l'islam et la qualité insuffisante des informations disponibles sur ce sujet dans les médias. Cette prise de conscience s'accentue fortement après les attentats de 2001. Une enquête menée auprès des lecteurs et de l'équipe du média ${ }^{57}$ démontre une volonté de produire une information dont la fiabilité

54. Ibid., p. 243-244.

55. Ibid., p. 244.

56. Ce qui n'exclut évidemment pas les rapports de pouvoir, mais les rapports sociaux ne s'y réduisent pas.

57. A.-S. LAmine, «Média minoritaire, diversité intra-religieuse et espace public. Analyse du site Saphirnews.com», Sociologie, no 2015/2, p. 139-156: https://www.cairn.info/revuesociologie-2015-2-page-139.htm [consulté le 17 juin 2020]. 
sera reconnue par son public cible, mais aussi par la profession journalistique («être des journalistes comme les autres») et par des lecteurs non musulmans. Il vise donc la neutralité et affirme le primat de la compétence professionnelle, et doit, à ce titre, être qualifié de média spécialisé plutôt que de média communautaire. Plusieurs indices démontrent d'ailleurs cette reconnaissance professionnelle: carte de presse, partenariats avec CrossCheck, invitation à des débats, articles cités ou retweetés par des journalistes de médias généralistes. Alors que nombre d'espaces militants sont dans une logique contre-hégémonique (et donc de différenciation), ce n'est pas le cas d'un tel média ${ }^{58}$. Il relève au contraire d'une démarche de dé-différenciation et de spécialisation, et donc d'une logique de participation au monde commun à partir d'une identité particulière.

L'étude du cas d'une mère musulmane nourrit le second questionnement. À l'invitation d'une déléguée de parents d'élèves, Nadia se porte bénévole pour aider à la préparation du goûter de Noël de l'école élémentaire de ses enfants ${ }^{59}$. Sachant que dans une autre école de la ville, une autre mère avait été écartée d'une sortie scolaire en raison de son foulard, elle prend les devants et va voir la directrice, "pour lui demander si [son] voile poserait problème», la réponse est "pas de voile ou alors pas de goûter». Une pétition de soutien circule, signée par des mères musulmanes et non musulmanes. La directrice annule le grand goûter devant rassembler tous les enfants et des parents, en le remplaçant par un petit goûter dans chaque classe, donc sans parents. Soulignant qu'elle veut « [s']investir dans la vie des établissements [que ses enfants] fréquentent», Nadia confie qu'elle a "vécu cette affaire dans la souffrance». En décembre 2013, une directive de l'inspecteur académique confirme l'interdiction de participer à des sorties scolaires aux mères voilées de Méru (Oise). Quelques mois plus tard, un groupe de mères se mobilise et porte l'affaire devant le tribunal administratif d'Amiens, qui leur donnera raison deux ans plus tard ${ }^{60}$. De tels cas sont fréquents et leurs issues variables.

Plusieurs enquêtes font état des réactions et mobilisations face à cette interdiction ${ }^{61}$. Ces femmes affirment sans ambiguïté leur appartenance iden-

58. En cela, il se différencie d'autres médias musulmans en ligne, plus engagés et militants (Al-Kanz.org, Islametinfo.fr, Ajib.fr...). Il ne faudrait pourtant pas en déduire qu'ils ne participent pas au monde commun, mais avec un processus de différenciation et une «inclusion négociée» (\$ 1.4. et note 25).

59. E. GARDET, «Une maman privée de goûter à cause de son voile», Le Parisien, 11 déc. 2013.

60. «Sorties scolaires à Méru: les mères voilées ont eu gain de cause », Le Parisien, 30 déc. 2015.

61. A. Kassir, J. G. Reitz, «Protesting Headscarf Ban: a Path to Becoming More French? A Case Study of "Mamans toutes égales" and "Sorties scolaires avec nous" ", Ethnic and 
titaire multiple en se référant à leur francité, à leur citoyenneté, ainsi qu'aux valeurs de tolérance et de vivre ensemble; elles réfutent fermement l'unique assignation religieuse. Elles définissent d'ailleurs leur mouvement comme un combat pour des droits civiques et non religieux, et ne recherchent aucunement le soutien de mosquées ou d'imams. Par ailleurs, elles expriment leur inquiétude quant aux effets de telles interdictions sur leurs enfants. Certaines inventent même des prétextes pour leur cacher qu'elles n'ont plus le droit d'accompagner les sorties, de crainte que cette situation ne donne à ceux-ci une mauvaise image de l'école et de la société. Il est avéré que l'effet de mésestime sociale impacte les mères; il touche aussi forcément au moins une partie de leurs enfants.

La posture d'interdiction - s'appuyant sur la circulaire Chatel de 2012, donc sur une lecture extensive de la loi de 2004, qui pourtant s'applique aux élèves et non aux parents - relève d'une posture républicaine selon laquelle la personne doit montrer qu'elle est une bonne citoyenne (selon les critères républicains d'invisibilité du religieux) avant d'être autorisée à participer à la construction du bien commun (ici la sociabilité dans et autour de l'établissement scolaire, mais aussi, dans le cas du goûter de Noël, le pluralisme en acte $\left.^{62}\right)$.

\section{CONCLUSION}

Même si, du point de vue religieux, les «minoritaires» (donc ceux qui ne sont ni catholiques ni sans religion) ne dépassent pas $10 \%$ de la population française, leur visibilité est accentuée par le fait qu'ils ont, en moyenne, une religiosité plus élevée que les catholiques. La comparaison des groupes permet de désingulariser le cas de l'islam à cet égard. De plus, lorsque l'on enquête sur ces «minoritaires», le risque est de se focaliser sur des discours et des principes, au détriment de ce que les gens font.

Concernant les identités religieuses, et à l'encontre de la vision de la religiosité comme une appartenance et une adhésion inconditionnelle et intransigeante à un dogme, à une communauté et à des règles de conduite, l'analyse pragmatiste offre une grille qui permet d'être attentif à ce que les

Racial Studies, 39/15, 2016, p. 2683-2700; H. Karimi, «The Hijab and Work: Female Entrepreneurship in Response to Islamophobia», International Journal of Politics, Culture, and Society, 31/4, 2018, p. 421-435.

62. Dans d'autres cas, c'est de contribuer à l'éducation à la citoyenneté par la visite, par exemple, d'un conseil départemental. 
gens font réellement: leurs pratiques, leurs ajustements, leurs relations aux autres et la pluralité de leurs systèmes de référence.

Enfin, dans cette perspective, il semble pertinent d'analyser l'évolution de l'intégration d'abord en termes de participation sociale (ce qui se fait) et de ne pas se limiter aux discours et aux signes extérieurs d'appartenance religieuse. Cette approche permet de comprendre que les processus de différenciation côtoient ceux de dé-différenciation, caractérisés par la fabrication du commun à partir d'identités particulières, d'être attentif à la multitude des formes que prend cette participation et, a contrario, de s'interroger sur les effets collatéraux des formes d'empêchements à la participation sociale ${ }^{63}$.

63. Outre l'effet déjà mentionné sur les mères et leurs enfants, on peut penser à l'effet discriminatoire en terme professionnel, M. Doytcheva, «Aux limites de la diversité, la religion: un angle mort des politiques de lutte contre les discriminations ", Confluences Méditerranée, no 3, 2018, p. 109-120. Ou encore aux effets collatéraux de la loi de 2004. À partir d'une analyse secondaire de deux grandes enquêtes, Emploi et Trajectoires et Origine ( $\mathrm{TeO})$, une étude se focalise sur les jeunes femmes en âge d'être impactées par la loi de 2004 au moment de leurs années de lycée et démontre une probabilité moindre d'obtenir le baccalauréat, et une probabilité plus grande de ne pas avoir d'activité professionnelle: A. Abdelgadir, V. Fouka, «Political Secularism and Muslim Integration in the West: Assessing the Effects of the French Headscarf Ban», Stanford University Working Paper, 2019: https://pdfs.semanticscholar.org/f90e/5de465d750c0fe020ccfbdd8118fobf 076b9.pdf [consulté le 17 juin 2020]. 